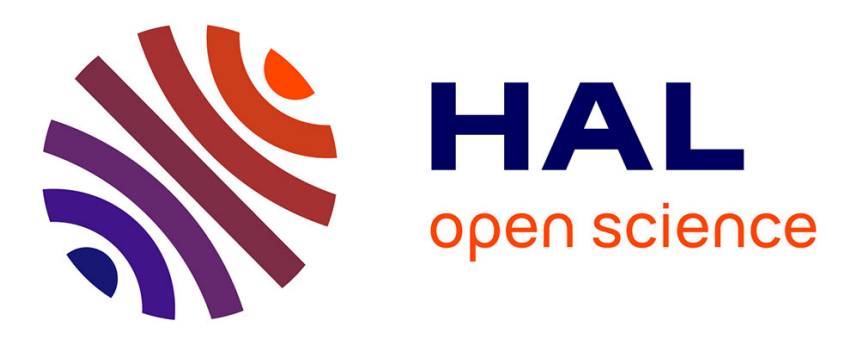

\title{
Power Efficiency of the Hybrid Broadcast Unicast Network with Suitable Resource Allocation
}

\author{
Ahmad Shokair, Matthieu Crussière, Jean-François Hélard, Oussama Bazzi,
} Youssef Nasser

\section{To cite this version:}

Ahmad Shokair, Matthieu Crussière, Jean-François Hélard, Oussama Bazzi, Youssef Nasser. Power Efficiency of the Hybrid Broadcast Unicast Network with Suitable Resource Allocation. IEEE International Symposium on Broadband Multimedia Systems and Broadcasting (BMSB 2018), Jun 2018, Valence, Spain. 10.1109/bmsb.2018.8436840 . hal-01819487

\section{HAL Id: hal-01819487 \\ https://hal.science/hal-01819487}

Submitted on 20 Jun 2018

HAL is a multi-disciplinary open access archive for the deposit and dissemination of scientific research documents, whether they are published or not. The documents may come from teaching and research institutions in France or abroad, or from public or private research centers.
L'archive ouverte pluridisciplinaire HAL, est destinée au dépôt et à la diffusion de documents scientifiques de niveau recherche, publiés ou non, émanant des établissements d'enseignement et de recherche français ou étrangers, des laboratoires publics ou privés. 


\title{
Power Efficiency of the Hybrid Broadcast Unicast Network with Suitable Resource Allocation
}

\author{
Ahmad Shokair ${ }^{* \dagger}$, Matthieu Crussière*, Jean-Francois Hélard*, Oussama Bazzi ${ }^{\dagger}$ and Youssef Nasser ${ }^{\ddagger}$ \\ * Univ Rennes, INSA Rennes, CNRS, IETR-UMR6164, F-Rennes, France \\ \{ashokair, mcrussie, jfhelard\} @insa-rennes.fr \\ $\ddagger$ American University of Beirut, Beirut, Lebanon 11072020 , \\ yn10@aub.edu.lb \\ † Lebanese University, Faculty of Sciences 1, Hadath, Lebanon, \\ obazzi@ul.edu.lb
}

\begin{abstract}
The demand on linear services like mobile TV has been growing during the last few years, creating challenges to the conventional methods of delivery such as broadcast networks and broadband mobile networks. In this paper, a hybrid approach with the coexistence of a broadcast transmitter and a unicast broadband transmitter is discussed, and the problem of the power consumption and power efficiency is interpreted. To achieve an efficient hybrid network in terms of power consumed to serve a user, the hybrid network is numerically optimized in terms of key design parameters like the broadcast radius and the base stations' density. The effect of user density is also studied highlighting the gain expected when using the hybrid approach. Results have shown that an optimal operation point could be found, and that the gain provided by the hybrid network depends on the density of the users.
\end{abstract}

Index Terms-Mobile TV, broadcast networks, broadband networks, hybrid networks, green communications, power efficiency.

\section{INTRODUCTION}

A High demand for mobile TV has been witnessed in the recent years following the increasing availability of handheld devices like smartphones and tablets [1]. Conventionally, mobile TV is delivered by either a dedicated high power Broadcast (BC) network like DVB-T2 and ATSC 3.0 [2], [3], or by utilizing the existing broadband networks like LTE. However, the limited spectral resources of Unicast (UC) broadband networks and constrained availability of BC networks in all zones led to the emergence of a new hybrid approach consisting in the coexistence of both broadcast and unicast networks. A hybrid network could be seen as an extension of the $\mathrm{BC}$ coverage, or an offload of traffic from $\mathrm{UC}$ to $\mathrm{BC}$ where necessary.

Hybrid networks can be categorized into two types, (a) same-area networks where both networks operate in the same area, and a user allocation scheme is used to assign users to either network like in [4], and (b) spatially separated networks, where users within a certain area around the broadcasting unit are served by $\mathrm{BC}$, and the rest are served by the broadband network like in [5]. In [6] Fam et al. studied the energy efficiency in this latter case of hybrid network using the grid model for the broadband subnetwork. In these studies, however, simple hybrid network models and resource allocation are used such that no interference occurs or is considered between the cells of the broadband subnetwork. The Authors presented in [7] a hybrid model with two scenarios of frequency allocation and derived analytical expressions for the probability of coverage and capacity, taking interference into consideration.

In this work, we present a model of a spatially separated hybrid broadcast/unicast network, using microcells for the broadband subnetwork, and taking inter-cell interference into consideration. Stochastic geometry tools with a Poisson Point Process (PPP) are used to model the broadband network. The resource allocation scheme proposed in [8] is adopted here to make the best use of spectral resources. We then analyze a set of design parameters affecting power efficiency of the hybrid network. The results leading to a power efficient hybrid network are provided and corresponding conclusions are drawn. The effect of those parameters on the optimal operating point is then studied.

In this work, we study the power energy efficiency for a user-dense spatially separated hybrid network, using microcells for the broadband subnetwork, taking inter-cell interference into consideration, utilizing stochastic geometry tools with a Poisson Point Process (PPP) to model the broadband network. The resource allocation scheme is proposed in [8] and adopted here on top of the stochastic geometry approach to design a power efficient hybrid network. The problem is set to minimize the power consumption per served user by managing the key design parameters like $\mathrm{BC}$ radius and $\mathrm{UC}$ BS's density. Numerical simulations are then done to solve this problem and find the optimum values of those parameters, and to study the effect of user density on the gain the hybrid approach provides.

The rest of this paper is organized as follows: Section II presents the model used for the hybrid network and Section III discusses the problem in hand and the metric that is used for optimization. Numerical simulation for the optimization of the power efficiency and the study of the effect of user density is presented in IV, and V concludes the work.

\section{NETWORK MODEL}

The hybrid model aims to serve $M$ users with linear services such as mobile TV. Those users are uniformly distributed over 
a circular service area with radius $r_{m} a x$. As shown in Fig.1, the circular service area is divided into a BC area and a UC area. The $\mathrm{BC}$ area is with radius $r_{b}$ and a High Power High Tower (HPHT) station is at the center serving users. Any user within the $\mathrm{BC}$ area is served by the HPHT. The UC area is the area not covered by $\mathrm{BC}$ where a user is served by the nearest broadband Base Station (BS), creating a Voronoi tessellation. The BSs are distributed according to PPP $\Phi$ with density $\lambda_{B S}$, and the users are distributed uniformly according to another PPP $\Psi$ with density $\lambda_{u}$. The HPHT is transmitting at frequency $f_{B}$ with power $P_{B}$, and the UC BSs outside the $\mathrm{BC}$ area are transmitting at the same frequency $f_{B}$ with power $P_{U}$ such that $P_{B}>P_{U}$. Using the same frequency band creates extra interference, but reduces the total occupied bandwidth (BW) by the system. Note that the regular UC BSs within the $\mathrm{BC}$ area are not serving linear services and are operating at a different frequency band, so they will not cause any interference with the rest of the system, and thus will not be further considered in this work.

\section{A. Signal to Interference and Noise Ratio}

The Signal to Interference and Noise Ratio (SINR) for BC users could be expressed as following:

$$
\begin{aligned}
\operatorname{SINR}_{B C} & =\frac{P_{r}}{P_{n}+I_{u c / b c}} \\
& =\frac{P_{B} g r_{v}^{-} \beta}{\sigma^{2}+I_{u c / b c}}
\end{aligned}
$$

where $P_{r}$ and $P_{n}$ represent the received power and noise power respectively, $I_{u c / b c}$ denotes the interference from UC BS on $\mathrm{BC}$ users, $g$ is an exponentially distributed random variable modeling the $\mathrm{BC}$ and random channel effect, $r_{v}$ denotes the distance between a $\mathrm{BC}$ user and the $\mathrm{BC}$ transmitter, and $\beta$ denotes the pathloss exponent for BC transmission. Similarly, SINR for the broadband users is given by:

$$
\begin{aligned}
\operatorname{SINR}_{U C} & =\frac{P_{r}}{P_{n}+I_{b c / u c}+I_{u c / u c}} \\
& =\frac{P_{U} h r_{l}^{-} \beta}{\sigma^{2}+I_{u c / b c}}
\end{aligned}
$$

where $I_{b c / u c}$ and $I_{u c / u c}$ denotes the interference from the BC transmitter on UC users and from the unicast BS on UC users respectively, $h$ is an exponentially distributed random variable that represent UC random channel effect, and $r_{l}$ is the distance between a user in the UC area and its serving BS, and $\alpha$ denotes the pathloss exponent for UC transmission. The interference relations are as following:

$$
\begin{gathered}
I_{u c / b c}=\sum_{j \in \Phi} P_{L} h r_{s, j}^{-\alpha} \\
I_{u c / u c}=\sum_{j \in \Phi / b} P_{L} h r_{q, j}^{-\alpha} \\
I_{b c / u c}=B_{R} P_{D} g r_{d}^{-\beta}
\end{gathered}
$$

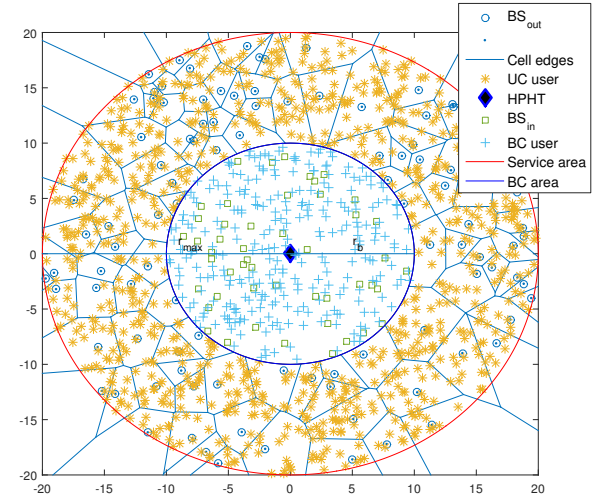

Fig. 1: An example of a hybrid network, with $20 \mathrm{~km}$ radius service area, including $10 \mathrm{~km} \mathrm{BC}$ radius

where $r_{s, j}$ is the distance between a BC user and interfering BS $j$ and $r_{q, j}$ is the distance from a UC user to an interfering $\mathrm{BS} j . B_{R}$ represent the $\mathrm{BW}$ ratio between the $\mathrm{UC}$ and the $\mathrm{BC}$.

\section{B. Resource allocation scheme}

Resource allocation is a key element in the implementation and operation of a mobile network. Several schemes have been used in the literature. However, non was originally designed for linear services. In this work, the algorithm we proposed in [8] is used for allocating resource blocks to users served by UC.

The idea behind this algorithm is to allocate resource blocks to a user enough to slightly exceed a certain threshold capacity $C^{r e q}$. The scheme is based on two concepts: start with users that have better network conditions, since those need fewer resources to be satisfied, and for each user start allocating the best resource blocks available so it can be satisfied with the lowest possible number of resource blocks. The algorithm showed near-optimal results maximizing the success rate (SR), and outperformed conventional resource allocation schemes like round robin, maximum throughput, and proportional fair. Note that the SR is defined as the ratio of the served users to all users in the service area.

Stochastic geometry introduces the PPP as a suitable form for modeling the positions of broadband network BS. It was shown in earlier studies that a countable difference exists between the SR achieved using the less accurate grid model and the SR achieved when a PPP is used [9]. A more accurate $\mathrm{SR}$ is very important in the evaluation of the power efficiency in the next section.

\section{Power Consumption: Problem Statement and SOLUTION}

In this section the power consumption model is explained, and the power efficiency metric is then discussed.

\section{A. Power consumption model}

It was shown in [10] that the power consumption of an LTE BS could be expressed as following:

$$
P_{\text {in }}=N_{T R X}\left(P_{0}+\Delta_{p} P_{\text {out }}\right)
$$


where $P_{\text {out }}$ is the output power of the power amplifier, $P_{0}$ represents the power consumption with no power transmitted, and $\Delta_{p}$ is the load dependent power consumption slope. $N_{T R X}$ is the number of transmit/receive chains. The reference also provides a table of numerical values for those constants depending on the nature of the BS being a macro cell, micro cell, femto cell... In this work, we used the micro cell model for the UC BSs, with $N_{T R X}=2, P_{0}=56 \mathrm{~W}$, and $\Delta_{p}=2.6$. Due to the lack of published model for the power consumption of a broadcast HPHT, the same model is used but with the macro cell set of constants, i.e. $N_{T R X}=6, P_{0}=130 \mathrm{~W}$, and $\Delta_{p}=4.7$.

\section{B. Performance metric: Power efficiency}

In linear services transmission, the main goal is to serve as many users as possible, where the global capacity is a secondary goal, since the stream rate is controlled by the transmitter, and any additional capacity a user might have will not enhance the quality of service. A user is said to be connected if the capacity allocated to it exceeds certain threshold capacity $C^{r e q}$. Therefore, a power efficient network is the one that serves a large number of user with a low power consumption. This could be measured by the power for user factor, which is the ratio of the number of served users and the total power consumed by the network, and can be expressed as follows:

$$
P_{u s e r}=\frac{P_{i n}^{B C}+\sum P_{i n}^{U C}}{S^{B C} N^{B C}+S^{U C} N^{U C}}
$$

where $P_{i n}^{B C}$ is the power consumed by the HPHT, $P_{i n}^{U C}$ is the power consumed by each UC BS, $S^{B C}$ and $S^{U C}$ are the success rate of $\mathrm{BC}$ and $\mathrm{UC}$ networks respectively, and $N^{B C}$ and $N^{U C}$ are the number of users in the $\mathrm{BC}$ and $\mathrm{UC}$ areas respectively. Since it is assumed that all BSs are transmitting the same power, and following the uniform distribution of the users and BSs in the service area, substituting the number of users and Bs by their respective values will result in the following expression:

$$
P_{\text {user }}=\frac{P_{i n}^{B C}+\pi \lambda_{B S}\left(R_{\max }^{2}-r_{b}^{2}\right) P_{i n}^{U C}}{\pi \lambda_{u}\left(r_{b}^{2} S^{B C}+\left(R_{\max }^{2}-r_{b}^{2}\right) S^{U C}\right)}
$$

In this expression, the success rate for BC users, $S^{B C}$, can be seen as the probability of a user exceeding a certain threshold SINR. This probability was derived in our previous works, and it's complexity makes it difficult to use it in the optimization analytically. Moreover, the success rate for the UC users, $S^{U C}$, which was achieved by the linear service oriented resource allocation scheme, has no closed-form expression that derives it. But what is known is that both success rates are functions of the design parameters and system parameters.

The aim is to exploit and optimize the hybrid network from the power consumption point of view. It can be concluded from (8) that the two main design parameters that control the power efficiency are the BC radius $r_{b}$ and the UC BS density $\lambda_{B S}$. For instance, increasing $r_{b}$ reduces the numerator with a variable effect on the denominator depending on other constants, and increasing $\lambda_{B S}$ increases the numerator and the denominator with different rates. Due to the complexity of an analytical optimization of 8 , Numerical optimization, and interpretation are done next.

\section{Simulation AND Discussion}

In this section, numerical evaluation is done to find a set of design parameters that minimize the power consumption per user, and to study the effect of system parameters on the effectiveness of the hybrid network.

\section{A. Simulation setting}

An area of $40 \mathrm{~km}$ radius is considered for the numerical evaluation. both UC and BC networks uses $800 \mathrm{MHz}$ band for transmission, with $10 \mathrm{MHz} \mathrm{BW}$ for $\mathrm{BC}$ and $20 \mathrm{MHZ}$ total BW for UC, with total number of $100 \mathrm{RB}$ per BS. This transmitted power is $60 \mathrm{dBm}$ for $\mathrm{BC}$ and $38 \mathrm{dBm}$ for $\mathrm{UC}$. The default simulation setting are summarized in Table I.

TABLE I: Simulation setting

\begin{tabular}{||cc||}
\hline Parameter & Value \\
\hline$r_{\max }$ & $40 \mathrm{~km}$ \\
\hline$r_{b}$ & $20 \mathrm{~km}$ \\
\hline$P_{D}$ & $60 \mathrm{dBm}$ \\
\hline$P_{L}$ & $38 \mathrm{dBm}$ \\
\hline$B W_{B C}$ & $8 \mathrm{MHz}$ \\
\hline$B W_{U C}$ & $20 \mathrm{MHz}$ \\
\hline$\alpha$ & 3.4 \\
\hline$\beta$ & 3.2 \\
\hline$\sigma^{2}$ & $-100 \mathrm{dBm}$ \\
\hline Antenna Gain & $14.77 \mathrm{~dB}$ \\
\hline
\end{tabular}

\section{B. Optimization of the hybrid network}

Tow main design parameters are considered for optimization: the broadcast radius $r_{b}$ and the BS density $\lambda_{B S}$.

1) Broadcast radius: To find the optimal values for $r_{b}$, the power consumption per user is calculated for several values of user density and BS density. The results are shown in Fig. 2 for 2a: 5 users per BS, 2b: 20 users per BS, and 2c: 50 users per BS.

The results show that for the three cases, i.e. low, medium and high user density, an optimal point can be found that minimizes the power consumption per served user. This indicated that a hybrid approach introduces gain to the system in terms of power efficiency.

The other main indication is that the effect of the density of BSs depends on the density of the users. For low user density in $2 \mathrm{a}$, the effect of increasing the BS density on the optimal radius is limited, while it's effect becomes notable with a medium density like in $2 b$, and even more significant with high user density like in 2c. In general, adding more BSs to the system increases the optimal radius, and the rate of this increase depends on directly on the user density. In particular, it can be seen in the last case when the user density is high, increasing the $\mathrm{BC}$ contribution by increasing its radius enhances the power efficiency. 


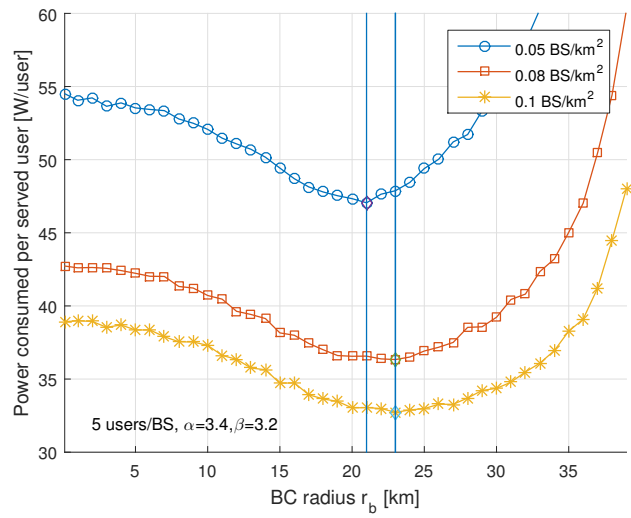

(a) 5 users per BS

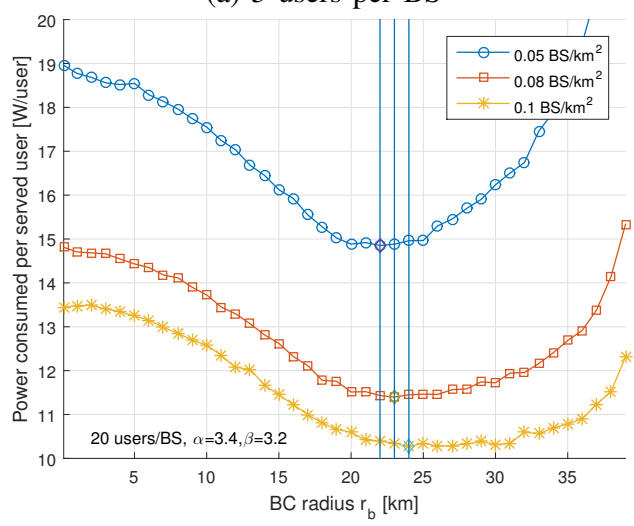

(b) 20 users per BS

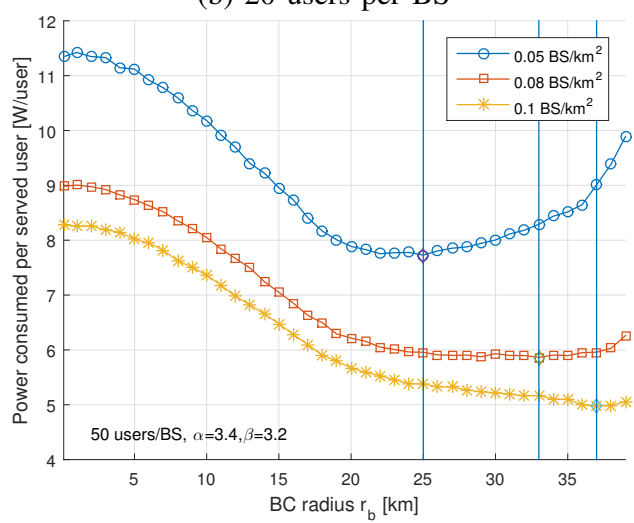

(c) 50 users per BS

Fig. 2: Power consumed per served user vs $r_{b}$

2) BS density: To highlight more the effect of UC BS's density, the power efficiency is evaluated for several values of $\lambda_{B S}$ for different user density cases. The results are shown in Fig. 3 and Fig. 4.

If the average number of users per BS is fixed, results in Fig. 3 show that increasing the number of BS enhances the power efficiency by reducing the power consumption per user. However, after a certain point, the increase in $\lambda_{B S}$ will not be as effective.

On the other hand, results in Fig. 4 show that if the number of users in the service area is fixed, increasing the BS density

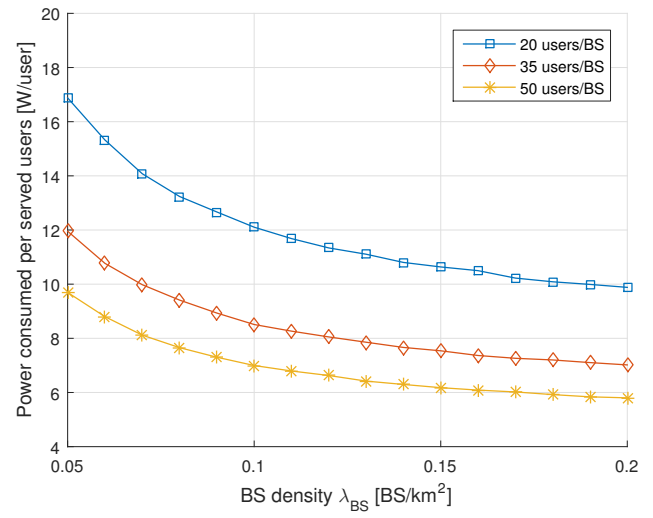

Fig. 3: Power efficiency for fixed user/BS ratio

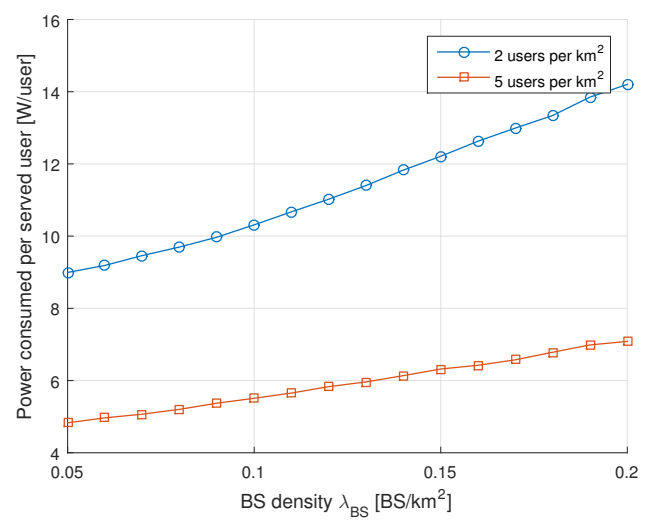

Fig. 4: Power efficiency for fixed number of users

will accumulate the power consumed per served user, and thus reduces the system power efficiency. The results also show that for low user density, the increase in power consumption per user is higher and faster. This is mainly due to the limited effect of the BS number increase on the success rate, with a large increase in the power cost.

\section{Effect of user density}

As can be seen from the results, the user density is a very important factor in the efficiency of the hybrid approach. Precisely, user density controls the success rate of the UC network, and consequently the number of connected users. It is worth mentioning that the resource allocation scheme contributed in reducing the effect of the huge increase in users by smartly allocating the available RB, and also reduced the power consumption by utilizing only a portion of the available RB. The system is studied under different values of user $\lambda_{u}$, and the results are shown in Fig. 5. Results show that, as expected, the power efficiency increases in a more dense area, where more users are served with an almost similar amount of power. To highlight the enhancement of the power efficiency provided by the hybrid approach for the different values of BS density and user density, The gain of the hybrid approach is studied. There exist two gain values, which are the power consumption drop caused by the hybrid approach compared to 
full BC network and full UC network. The results are shown in Fig.6.

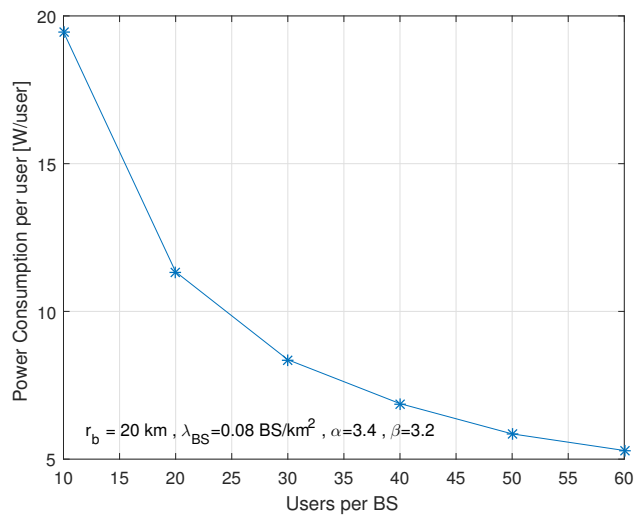

Fig. 5: Power consumed per served user vs user density

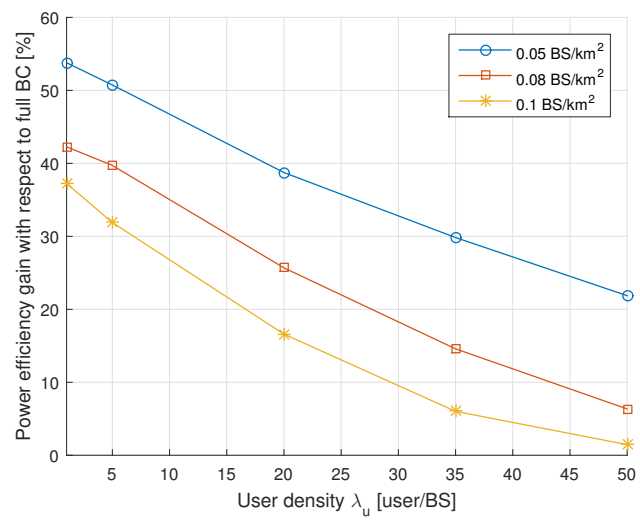

(a) Gain w.r.t. full BC

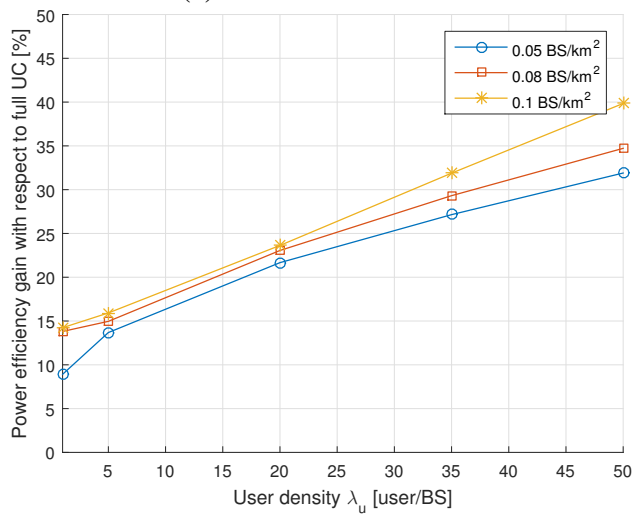

(b) Gain w.r.t. full UC

Fig. 6: Gain provided by hybrid network

Results show that compared to a full BC network, i.e. the case when $r_{b}=R_{\max }$, the highest gain a hybrid network can provide is when the user density is low. Under such conditions, a BC network has a relatively low efficiency, due to high transmission power, without having additional value due to the low number of users. On the other hand, compared to a full UC network, i.e. the case when the HPHT is removed, the maximum gain provided by the hybrid network is when the user density is the highest. This is mainly due to the fact that UC BSs have limited number of RBs to allocate, and thus a limited number of served users.

\section{CONCLUSION}

In this paper, a hybrid broadcast/unicast model for the delivery of mobile TV is studied, using stochastic geometry tools and micro BS in the UC subnetwork. The aim is to test the effectiveness of the hybrid approach in terms of power efficiency and to find the set of parameters that minimize the power consumption per served user. Results show that an optimal broadcast radius could be found and that the effect of the BS's density on that radius is related to the user density. Results also show that increasing the density of the BSs increases the efficiency of the system up to a certain level beyond which the efficiency is saturated. Moreover, it was shown that increasing the user density will decrease the efficiency of the system as expected. The results also show that the denser a network is, the highest the gain of a hybrid network will be compared to a full UC network while decreasing the density will increase the gain compared to a full BC network.

\section{ACKNOWLEDGMENT}

This work has received a French state support granted to the Convergence TV project through the $20^{\text {rd }}$ FUI (transverse inter-ministry funding) program. The authors would also like to thank the "Image \& Réseaux" and "Cap Digital" French business clusters for their support of this work.

\section{REFERENCES}

[1] Research and Markets, Global Mobile TV Market Size, Market Share Application Analysis, Regional Outlook, Growth Trends, Key Players, Competitive Strategies and Forecasts, 2017 to 2025, 2017.

[2] M. El-Hajjar and L. Hanzo, "A survey of digital television broadcast transmission techniques," IEEE Communications Surveys Tutorials, vol. 15, no. 4, pp. 1924-1949, 2013.

[3] L. Fay, L. Michael, D. Gmez-Barquero, N. Ammar, and M. W. Caldwell, "An overview of the atsc 3.0 physical layer specification," IEEE Transactions on Broadcasting, vol. 62, no. 1, pp. 159-171, 2016.

[4] C. Heuck, "An analytical approach for performance evaluation of hybrid (broadcast/mobile) networks," IEEE Transactions on Broadcasting, vol. 56, no. 1, pp. 9-18, 2010.

[5] A. A. Razzac, S. E. Elayoubi, T. Chahed, and B. El Hassan, "Planning of mobile tv service in standalone and cooperative dvb-ngh and lte networks," in WiOpt, 2013, pp. 609-614.

[6] P. A. Fam, S. Paquelet, M. Crussière, J. F. Hélard, and P. Bretillon, "On the energy efficiency of hybrid unicast-broadcast networks for mobile tv services," Journee Scientifique URSI-France 2016 Energie et Radiosciences, 2016.

[7] A. Shokair, Y. Nasser, O. Bazzi, J. F. Hélard, and M. Crussière, "On the coexistence of broadcast and unicast networks for the transmission of video services using stochastic geometry," submitted to IEEE Transactions on Broadcasting, 2018.

[8] A.Shokair, Y. Nasser, O. Bazzi, J.F. Hélard, and M. Crussière, "Near optimal linear-service oriented resource allocation strategy for lte networks," in ICUMT, 2017.

[9] A. Shokair, M. Crussiere, J.F. Helard, Y. Nasser, and O. Bazzi, "Mobile tv directed resource allocation scheme for lte networks," in Wireless Personal Multimedia Communications (WPMC), 2017 20th International Symposium on. IEEE, 2017, pp. 241-246.

[10] G. Auer, O. Blume, and V. Giannini, "Energy efficiency analysis of the reference systems, areas of improvements and target breakdown," Tech. Rep., EARTH PROJECT, 2010. 